\title{
An Acousto-Ultrasonics Pattern Recognition Approach for Damage Detection in Wind Turbine Structures
}

\author{
M. Anaya, D.A.Tibaduiza, E.Forero, R.Castro \\ Faculty of Electronics Engineering \\ Universidad Santo Tomás Colombia \\ diegotibaduiza@usantotomas. edu.co \\ maribelanaya@usantotomas.edu.co \\ edwinforero@usantotomas.edu.co \\ rafaelcastro@usantotomas.edu.co
}

\author{
F. Pozo \\ Department of Applied Mathematics III \\ CoDAlab, \\ Universitat Politécnica de Catalunya \\ francesc.pozodupc.edu
}

\begin{abstract}
The design of a Structural Health Monitoring (SHM) systems is a requirement in the task of improving the safety and maintainability of the structures. Among the multiple techniques available for health monitoring, acousto-ultrasonics $(A U)$ offers the possibility of inspecting large areas of structures from a piezoelectric active sensor network with a relatively small number of sensors. This paper proposes the use of an active piezoelectric sensor network which uses PZTs transducers working as sensors or actuators in different actuation phases to inspect a wind turbine structure to determine the presence of damages by analysing the data from the sensors with multivariate statistical methods such as PCA and damages indices in order to discern damages from the healthy structure.
\end{abstract}

\section{Introduction}

Continuous monitoring of all the components of a wind turbine is one of the application areas in the development of structural health monitoring systems (SHM) which is an important discipline that seeks to ensure the proper performance of a structure and security of service in its continuous operation. This objective can be achieved by different ways, however pattern recognition is one of the most common methods. In these approaches, a sensor network permanently attached to the structure is often used for inspecting and defining its current state based on the analysis of structural responses by means of computational algorithms [4]. As a result, the collected structural responses are analysed and compared with baseline patterns in order to detect abnormal characteristics and define the structural integrity. The obtained information can be used to define when the structure can operate and under which conditions $[8,12]$.

Structural Health Monitoring considers four levels in the damage diagnosis process [7]. The main objective in the first level is to know whether there is any abnormality in the structure and if this abnormality corresponds to damage. In the second level the damage localization allows determining the position of the damage in the structure. Third level includes classification tasks to define the type of damage and its size. Finally, the level 4 is focused on knowing the structure remaining lifetime. This paper is addressed to the first level of the damage identification task by means of a methodology which uses a piezoelectric active system, multiway principal component analysis (MPCA) and statistical damage indices to solve the damage detection problem. The approach is tested in a wind turbine blade which is instrumented with four piezoelectric transducers.

This work is organized as follows: Section 2 includes a brief theoretical background with the basic information about the different methods used in the methodology applied. Section 3 shows the experimental setup and describes the methodology used to inspect and diagnose the structure. Later, Section 4 includes the experimental results for the detection when just two scores are used. The need for the damage indices for the damage detection is also justified in this Section. Finally, Section 5 presents the conclusions.

\section{Theoretical background}

\subsection{Principal Component Analysis (PCA)}

Principal component analysis (PCA) is a classical method used in multivariate statistical analysis with the goal of dimensionality reduction in order to obtain data reduction 
and, more precisely, feature extraction. It was developed by Karl Pearson in 1901 and integrated to the mathematical statistics in 1933 by Harold Hotelling [5]. The general idea in the use of PCA is to find a smaller set of variables with less redundancy $[3,6]$. To find these variables, the analysis includes the transformation of the current coordinate space to a new space to re-express the original data trying to filtering the noise and redundancies. These redundancies are measured by means of the correlation between the variables. The application of PCA starts with a matrix $\mathbf{X}$ which contains information from $m$ sensors and $n$ experimental trials [8]. The data collected from the active sensor network are normalized by using the scale procedure called group scaling [11]. This pre-processing considers changes between sensors and does not process them independently. Since the scaled matrix $\mathbf{X}$ is a mean-centered matrix, it is possible to calculate the covariance matrix as follows:

$$
\mathbf{C}_{\mathbf{X}}=\frac{1}{n-1} \mathbf{X}^{T} \mathbf{X}
$$

The covariance matrix $\mathbf{C}_{\mathbf{X}}$ is a symmetric matrix that measures the degree of linear relationship within the data set between all possible pairs of variables (sensors). The subspaces in PCA are defined by the eigenvectors and eigenvalues of the covariance matrix as follows:

$$
\mathbf{C}_{\mathbf{X}} \mathbf{P}=\mathbf{P} \Lambda
$$

where the diagonal terms of matrix $\Lambda$ are the eigenvalues $\lambda_{i}, i=1, \ldots, N \cdot L$, of $\mathbf{C}_{\mathbf{X}}$ whereas the off-diagonal terms are zero. While the columns of $\mathbf{P}$ are the eigenvectors of $\mathbf{C}_{\mathbf{X}}$. This eigenvectors are classified according to the eigenvalues in descending order and they are called the principal components or the loading vectors of the data set. The eigenvector with the highest eigenvalue represents the most important pattern in the data with the largest quantity of information [9].

\subsection{Damage detection indices based on PCA}

Several damage detection indices based on PCA have been proposed and applied with excellent results in pattern recognition applications. In particular, two damage indices are commonly used: (i) the $Q$ index (also known as SPE, square prediction error) and (ii) the Hotelling's $T^{2}$ index [2].

The $Q$ index of the $i$ th experimental trial $x_{i}^{T}$ measures the magnitude of the vector $\tilde{x}_{i}^{T}:=\tilde{\mathbf{X}}(i,:)$, that is, the events that are not explained by the model of principal components [10], and it is defined as follows:

$$
Q_{i}=\tilde{\mathbf{X}}(i,:) \tilde{\mathbf{X}}(i,:)^{T}=x_{i}^{T}\left(\mathbf{I}-\hat{\mathbf{P}} \hat{\mathbf{P}}^{T}\right) x_{i}
$$

The $T^{2}$ index of the $i$ th experimental trial $x_{i}^{T}$ is the weighted norm of the projected vector $\hat{t}_{i}^{T}:=\hat{\mathbf{T}}(i,:)=$ $x_{i}^{T} \hat{\mathbf{P}}$, that is, a measure of the variation of each sample within the PCA model and it is defined as follows:

$$
T_{i}^{2}=\sum_{j=1}^{\ell} \frac{\hat{t}_{i, j}^{2}}{\lambda_{j}}=\hat{t}_{i}^{T} \Lambda^{-1} \hat{t}_{i}=x_{i}^{T}\left(\hat{\mathbf{P}} \Lambda^{-1} \hat{\mathbf{P}}^{T}\right) x_{i}
$$

\section{Experimental setup and methodology}

The experimental setup consists of a blade from a wind turbine which is instrumented with an active piezoelectric system that works in several actuation phases [1]. The wind turbine is a vertical axis wind generator ALEKO WGV75W which is designed to produce $50 \mathrm{~W}$ as nominal power and its maximum power is $75 \mathrm{~W}$ with 24 volts in direct current (see Figure 1). The speed feature of this wind generator for nominal power is 10 meters per second but is operates from $3 \mathrm{~m} / \mathrm{s}$ to $20 \mathrm{~m} / \mathrm{s}$ however the survival wind speed is $35 \mathrm{~m} / \mathrm{s}$ where the SHM system become very important to detect and alert for damage $i$ the structure. The wind generator has a full weight of $10.5 \mathrm{~kg}$, its rotor diameter is $560 \mathrm{~mm}$, it has five blades made in aluminum alloy with a length of $745 \mathrm{~mm}$. Figures 1 and 2 show the wind turbine and the blade.

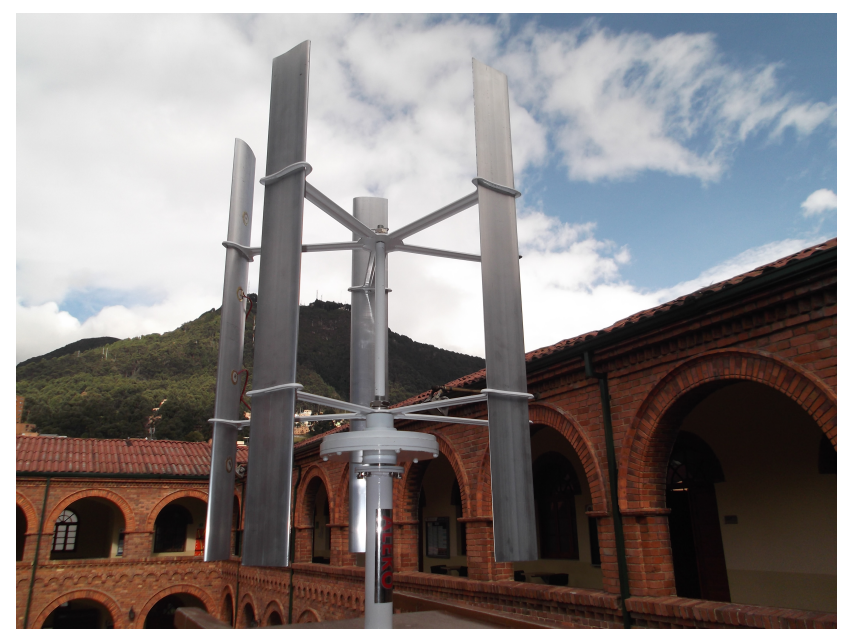

Figure 1. Vertical axis wind generator ALEKO WGV75W

The inspected blade was instrumented by means of 4 PZT transducers which are distributed over one face as it is shown in figure 2. As excitation input, a BURST signal with $1 \mathrm{Mhz}$ as central frequency, 5 peaks and 8 Volts of amplitude was used. Eight different states of the structure were analyzed: a healthy state and seven damages. Damages were simulated by adding a mass at seven different positions of the structure (see Figure 3). 


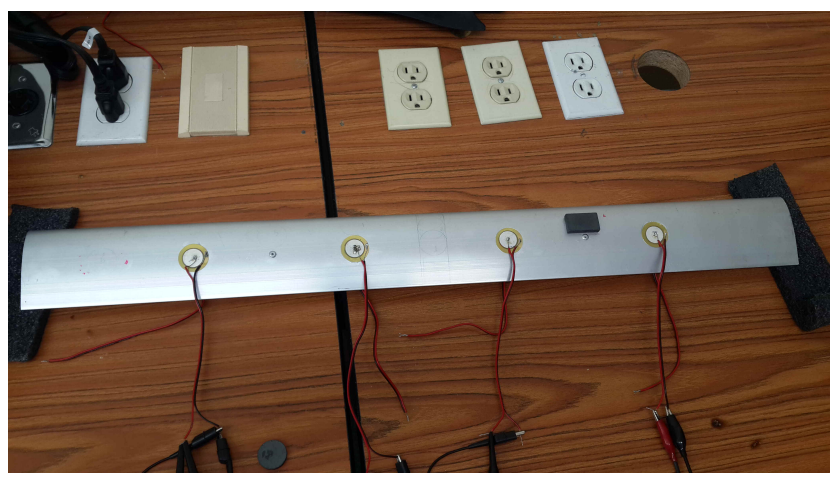

Figure 2. Instrumented Blade

40 experiments for healthy state and 20 experiments per damage were performed and collected. The acquisition modules are two TiePie HS4 oscilloscopes and the arbitrary signal generator is a TiePie HS5.

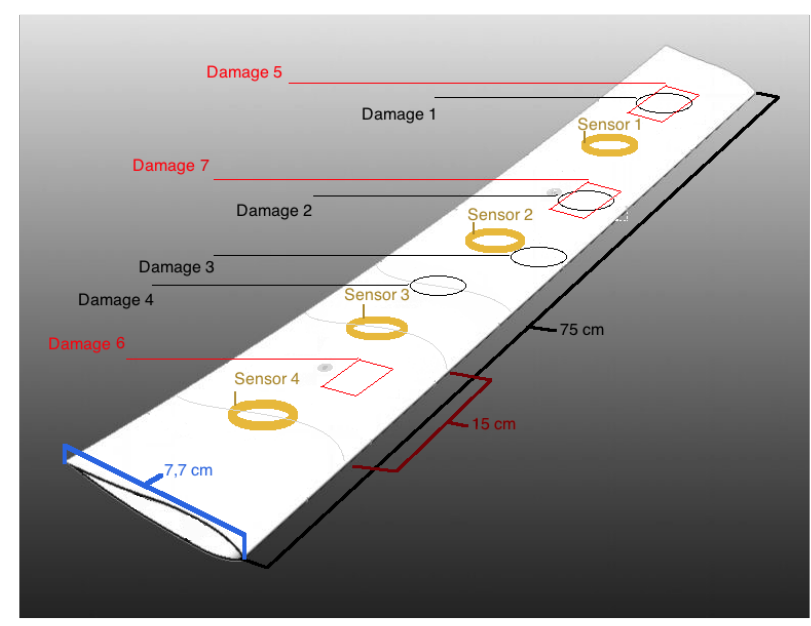

Figure 3. Instrumented Blade

The methodology applied in this work involves the use of a piezoelectric system which is used to inspect the structure in different actuation phases using a distributed piezoelectric active networks attached to its surface. In each actuation phase a PZT transducer is used as actuator by applying a signal excitation. Then, the propagated signal through the structure is collected by using the PZT transducers that work as sensors. This procedure is repeated with all the PZT transducers attached to the structure in order to obtain information from all the structure.

The data collected from each actuation phase are stored by using a data acquisition system in a 3D matrix
$(I \times K \times J)$, however this matrix is unfolded in a 2D matrix $(I \times K J)$ where $I$ represents the quantity of experiments, $K$ represents the number of samples times and $J$ represents the number of transducers (PZT) that act as sensor in the actuation phase. This unfolding process is illustrated in Figure 4. Since the data in each matrix come from experimental trials and could have different magnitudes and scales, it is necessary to apply a preprocessing step to scale the data using the mean of all measurements of the sensor at the same time and the standard deviation of all measurements of the sensor. This task is performed by applying group scaling normalization [1].

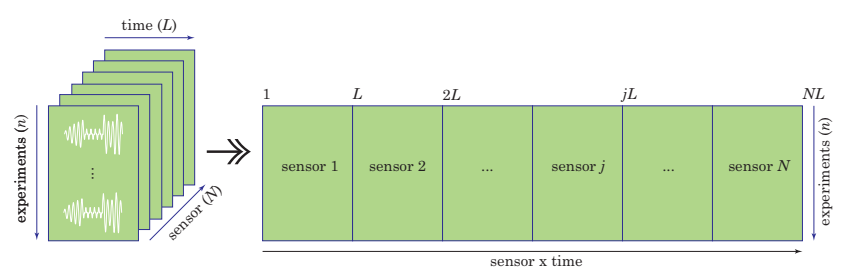

Figure 4. Unfolding the collected data in three dimensions to a two-dimensional matrix (I X JK) [8]

Using the data from the healthy structure a PCA model is built by each actuation phase. PCA was chosen by its utility in multivariate analysis. The PCA model is built by using 15 principals components. The data from different structural states are projected into each PCA model in order to obtain the scores and for calculating the damage indices, in his way, the $T^{2}$-index and $Q$-index obtained from each experiment in each actuation phase are used to detect the different damages.

\section{Experimental results}

Figures 5 and 6 shows the results obtained in the actuation phases 1 and 3 when two scores are ploted, this means when the PZT 1 and PZT 3 are working as actuators and the rest of the PZTs are used as sensors as it was explained in the previous section.

In a previous work by the authors [8], it was demonstrated that the retained variance by each component influence the results in the detection, this means that just two components give good results when a big percentage of variance is included. From the results in these figures, it is possible to conclude that is impossible to identify the undamaged data and cannot be applied the damage detection, this is because the data from all the structural states is mixed. 


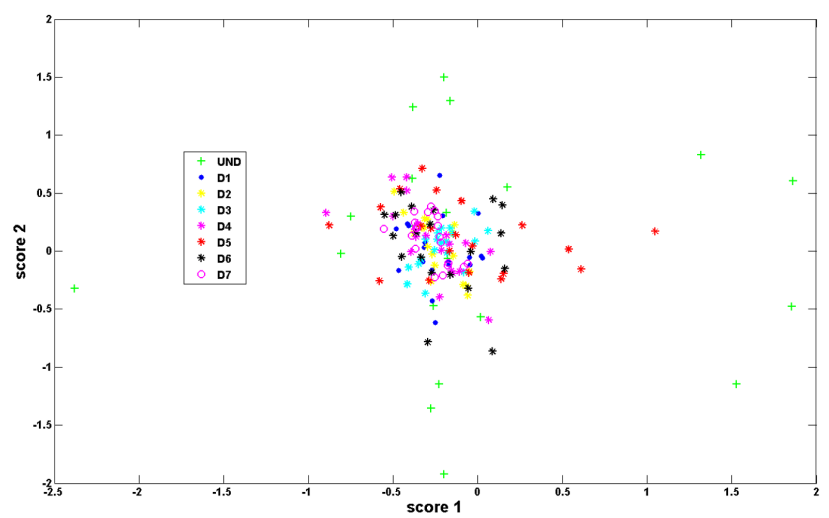

Figure 5. Score 1 vs Score 2 in the actuation phase 1

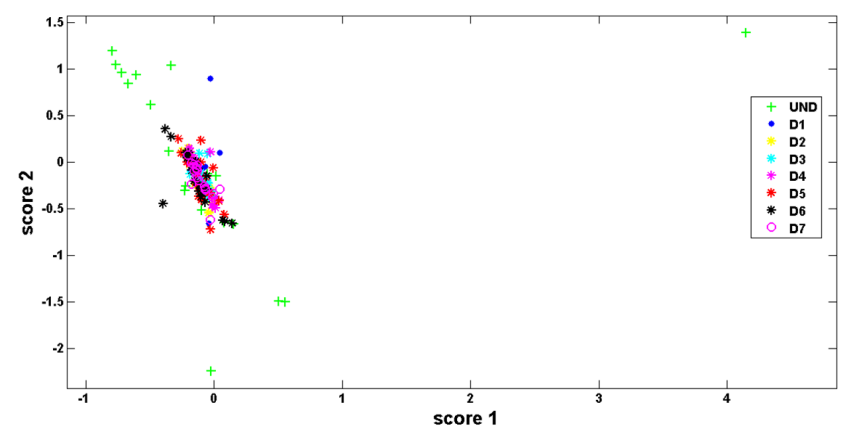

Figure 6. Score 1 vs Score 2 in the actuation phase 3

To solve this problem the damage indices are computed, in this case the Figures 7 and 8 show the results when $Q$ vs $T^{2}$ is plotted in the actuation phases 1 and 3 . As it is shown, in this case the undamaged state can be identified and it is possible to identify the presence of damages.

Similar results are obtained with the rest of the actuation phases. As it is possible to conclude from the previous Figures, two scores are not enough to determine the presence of damages in the structure. This is because the data that represent the damage are mixed with the data from undamage or health state of the structure.

\section{Conclusions}

A damage detection methodology based on principal components and damage indices was implemented. The methodology shows that all the damages were detected and distinguished with the healthy state. However, comparing the results from the scores plots and the damage indices plots, the damages are not detected in the scores plot when only two scores are used to represent the data. This is

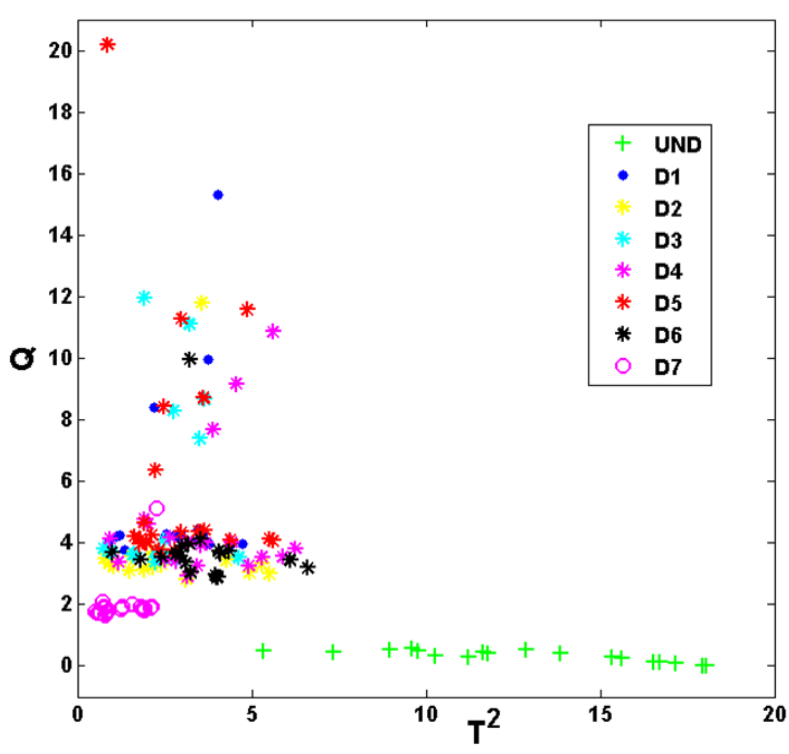

Figure 7. Index $T^{2}$ vs Index $Q$ in the actuation phase 1

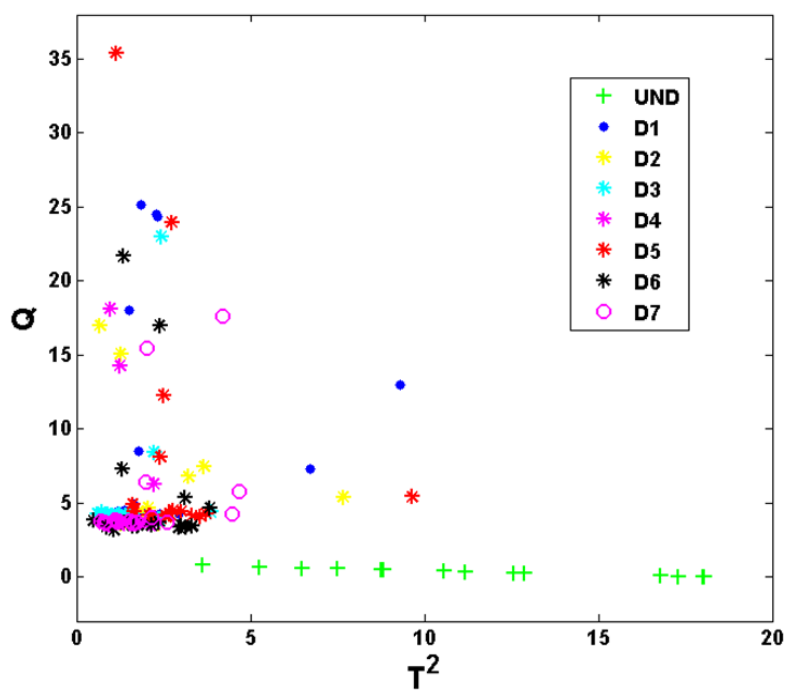

Figure 8 . Index $T^{2}$ vs Index $Q$ in the actuation phase 3

because, two scores do not represent the variability of the data. As A solution to this problem, the damage indices were applied using more scores allowing to improve the results in the detection process.

The use of the damage indices allowed to detect the presence of a damage, however it is not possible to discern the kind of damage. This is because the method is designed to compare with the healthy state as pattern.

Finally, it is necessary to remark that the use of the active piezoelectric system allowed to prove the PCA 
based methodology and validate the results from different actuation phases to identify a healthy structural state or the presence of damages at the surface by changing the PTZ used as actuator

\section{Acknowledgment}

This work is supported by Universidad Santo Tomás through Grant FODEIN 2015, project code 8410083003036.

\section{References}

[1] M. Anaya, D. Tibaduiza, and F. Pozo. A bioinspired methodology based on an artificial immune system for damage detection in structural health monitoring. Shock and Vibration, Article ID 648097, in press, 2015.

[2] A. Hot, G. Kerschen, E. Foltete, and S. Cogan. Detection and quantification of non-linear structural behavior using principal component analysis. Mechanical System and Signal Processing, 26:104-112, 2012.

[3] A. Hyvarinen, J. Kahunen, and E. Oja. Independent Component Analysis. John Wiley \& Sons, INC, 2001.

[4] T. Loutas, A. Panopoulou, D. Roulias, and V. Kostopoulos. Intelligent health monitoring of aerospace composite structures based on dynamic strain measurements. Expert Systems with Applications, 39:8412-8422, 2012.

[5] B. Mnassri, E. E. Adel, and M. Ouladsine. Fault localization using principal component analysis based on a new contribution to the squared prediction error. In 16th Mediterranean Conference on Control and Automation Congress Centre, ajaccio, France. June 25-27, 2008.

[6] L. Mujica, M. Ruiz, F. Pozo, J. Rodellar, and A. Güemes. A structural damage detection indicator based on principal component analysis and statistical hypothesis testing. Smart Materials and Structures, 23(2):025014, 2014.

[7] A. Rytter. Vibration Based Inspection of Civil Engineering Structures. PhD thesis, Department of Building Technology and Structural Engineering. Aalborg University, Denmark, 1993.

[8] D. Tibaduiza. Design and validation of a structural health monitoring system for aeronautical structures. PhD thesis, Department of Applied Mathematics III, Universitat Politcnica de Catalunya, 2013

[9] D. Tibaduiza, L. Mujica, M. Anaya, J. Rodellar, and A. Güemes. Principal component analysis vs independent component analysis for damage detection. In 6th EuropeanWorkshop on Structural Health Monitoring. DresdenGermany, July 2012.

[10] D. A. Tibaduiza, L. Mujica, J. Rodellar, and A. Güemes. Structural damage detection using principal component analysis and damage indices. Journal of Intelligent Material Systems and Structures, 2015.

[11] D. A. Tibaduiza, L. E. Mujica, and J. Rodellar. Damage classification in structural health monitoring using principal component analysis and self-organizing maps. Structural Control and Health Monitoring, 20:1303 - 1316, 2013.
[12] M. Torres, D. Tibaduiza, L. Mujica, J. Rodellar, and C. Fritzen. Data-driven multivariate algorithms for damage detection and classification: Evaluation and comparison. International Journal on Structural Health Monitoring, 13(1):19-32, January 2014. 